\title{
Modelling and Animating Hand Wrinkles
}

\author{
X. S. Yang and Jian J. Zhang* \\ National Centre for Computer Animation, \\ Bournemouth University, United Kingdom \\ \{xyang, jzhang\}@bournemouth.ac.uk
}

\begin{abstract}
Wrinkles are important visual features on the skin of an animated character. However, realistic animation of wrinkles is not easy, especially for the hand, as it gathers many types of different wrinkles. In this paper, we present a computationally efficient method for the generation of hand wrinkles, both for the palm and the fingers. This method is developed by exploiting the inherent properties relating to wrinkles, which are volume preservation of the underlying flesh and incompressibility of the skin. Although this method is for the hand model, it is also applicable to other parts of the body where the skin is deformed by underlying skeleton joints.
\end{abstract}

\section{Introduction}

Wrinkles are important visual features for realistic animated characters. On a human body, there are two types of wrinkles: fine-scale and large-scale ones. Between them, the latter is more significant in computer animation, as the largescale wrinkles mark pronounced feature lines on the skin surface and are obvious to the human eye. The large-scale wrinkles change with the motion of the character and capture the motion related skin deformations. The fine-scale ones are, on the other hand, much less visible and are relatively static compared with the other type.

There have been a number of techniques developed in representing and animating wrinkles on an animated human character. The fine-scale wrinkles are usually produced as texture on the skin mesh. As long as the skin area concerned does not deform to a large amount, this technique is effective and is reasonably realistic. Physiologically, large-scale wrinkles on a human body were there when the person was born, starting with shallow lines. These lines get deeper with the repetitive motion of the body parts, such as those on the forehead and the palm. Human skin deforms due to the properties of the skin and the underlying internal body elements. Distinctive skin deformation patterns are formed around wrinkles as a result of two most significant features relating to the human skin. The first is the incompressibility of the skin and the other is the volume preservation property of the underlying flesh. To animate a human character

\footnotetext{
* Corresponding author.
} 
realistically, these properties need to be convincingly modelled. This accounts for the difficulty for simulating large-scale wrinkles realistically in computer animation.

In this paper, we concentrate on the problem of large-scale wrinkle modelling, in particular, we will look into how hand wrinkles can be modelled and animated realistically within current animation production pipeline. Because of the properties of the skin around a wrinkle, physically simulating the deformation process is no doubt ideal. Nevertheless, such an approach often involves a sophisticated mechanical model and is likely to involve heavy computational overheads. Since what matters to animation is visual realism and in many case computational efficiency, in this paper we will develop a non-physically based simulation technique. The idea is to reproduce the skin deformation parametrically around large-scale wrinkles.

The remainder of the paper is structured as follows. Previous work was briefly reviewed in Section 2. Section 3 discusses the issues relating to a wrinkle deformation region including mesh generation. In Section 4.1, the shape of the cross section of large-scale wrinkles is derived. This is then followed by wrinkle animation in Section 4.2. Section 5 concludes the paper.

\section{Previous Work}

The most straight forward idea to generate wrinkles is texture mapping. The texture can be either extracted directly from photos or synthesized. However, it is difficult to animate, especially when large deformation is involved. Viaud and Yahia [1] presented one of the early methods for wrinkle modelling. They modelled wrinkle bulges as spline segments. Bando et al 2 presented a technique for the modelling of both fine-scale and large-scale wrinkles. For the fine-scale ones, their method is in essence a texture synthesis technique. For large-scale wrinkles, they displace the vertices concerned in the normal direction of the wrinkle shape. Adaptive mesh refinement is carried out for those areas if the mesh is too coarse for the required resolution. Larboulette and Cani 3 recently presented a technique for generating dynamic wrinkles geometrically. Their idea is to model wrinkles as a length preserving curve. When a curve is pushed at one end, wrinkles start to appear and ripple through the whole length. Because of this analogy, their method is effective on parallel wrinkles appearing on both cloth and human skin.

Physics-based techniques have also been studied by many researchers [4]. Wu's method [5] includes both texture mapping and a linear plastic model to simulate the wrinkling phenomenon. Boissieux et al. [6] modelled skin as a volumetric substance with a layered structure. The finite element method was used to compute the deformation. In general, physical modelling methods tends to produce mathematically more accurate results, however, are in practice more expensive than other alternatives. 


\section{Wrinkle Deformation Regions}

On the palm and other parts of the human body where large-scale wrinkles appear, the skin deforms locally when the relevant body parts move. In this Section, we present a method to define a deformation region for each or a group of wrinkle(s). We also discuss how this region is seamlessly merged with the existing surface mesh of the animated character model.

\subsection{Wrinkle Lines}

Wrinkle lines define the direction and pattern of the wrinkles. These lines are user-specified and can be either manually drawn or obtained from other techniques. Essentially, they reflect the intention of the user (animator). Once these lines are given, our technique will then produce the mesh geometry and deformation following the animation parameters. Figure 1 shows some typical wrinkle lines on the palm, each line consists of a number of segments.

Fig. 1. Hand-drawn wrinkle lines

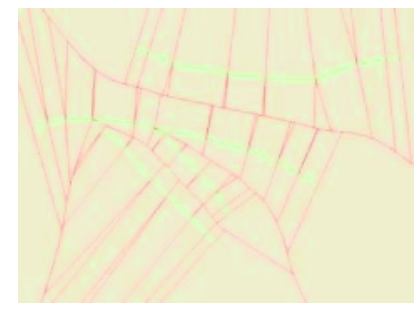

Fig. 2. Voronoi diagram and wrinkle lines

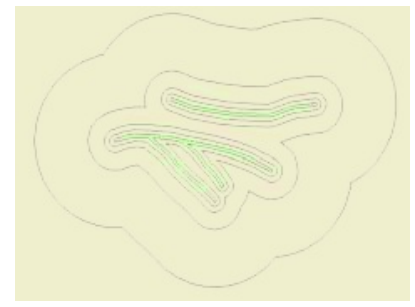

Fig. 3. Wrinkle lines and deformation region

\subsection{Determination of Wrinkle Deformation Regions}

Media axes are feature lines which can partition an irregular region with some sense of 'symmetry' [7. From observing skin deformation resulting from wrinkles, we believe it is reasonable that their deformable region can be defined as an area where the wrinkles are regarded as their media axes.

Practically, we try to define an offset curve for each wrinkle line on the parametric space of the existing model. As each wrinkle line is a separate line entity, its offset curve actually marks out a closed region. The overall deformation region for a group of connected and closely laid wrinkle lines are taken as the union of all the separate regions.

To compute the boundary and mesh of this region, we construct a Voronoi diagram. Algorithmically, for each wrinkle line, we have adopted the algorithm from [7]. Figure 2 shows the Voronoi diagram (red) corresponding to the wrinkle lines (green). By considering the influence distance, we are able to mark the deformation region out of the diagram, as shown in Figure 3. 


\subsection{Mesh Generation}

One of the advantages of using a wrinkle deformation region is that we can avoid mesh refinement, which can be expensive, as the original mesh usually does not guarantee that sufficient number of mesh vertices are placed within the wrinkle area. Mesh subdivision is necessary to produce a large number of densely populated vertices. This however, can be unnecessarily expensive. Using the deformation regions presented in above, the mesh is ready in place to deform the skin in a required manner.

By superimposing Figure 2 with Figure 3, we can generate the mesh in the deformation region, as seen in Figure 4. The vertices are in the right place to represent wrinkle deformation. From our experiment, this initial mesh is adequate for most wrinkle animation applications.

Now we are ready to merge the deformation region with the animation model, in our case, the hand model. This is a straightforward process. What we need to do is to place the deformation region on the parametric space of the model and remove all vertices falling into the region from the model. The deformation region carved out of the model mesh is patched up by the Voronoi mesh (Figure $5 \mathrm{a})$. At the boundary of the deformation region, vertices from the original model mesh and the Voronoi mesh may not match exactly. Triangulation around the boundary is necessary to fix it. Figure 5b shows the mesh of the wrinkle deformation region merged with the original hand mesh. Notice that the mesh does not need to be dense.

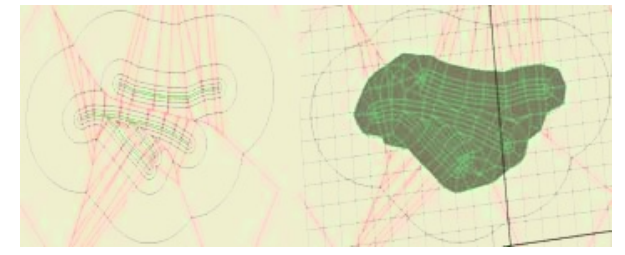

Fig. 4. Initial mesh in the deformation region

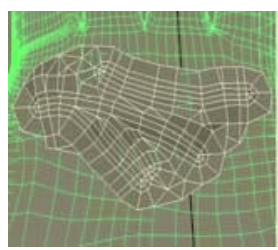

a

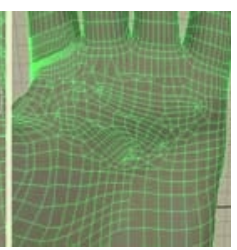

b

Fig. 5. Merge both meshes together

\section{Animation of Wrinkles}

The animation of wrinkles involves two key elements, deformation simulation conforming to the skin properties around wrinkles; and the establishment of the relationship between deformation and motion parameters.

\subsection{Skin Deformation}

Realistic skin deformation relies on the simulation process to recognise the properties of the skin around a wrinkle. As the visual quality is what we are after rather than the physical accuracy, we do not intend to simulate the phenomenon 
based on the physical principles that govern the deformation. Instead, we will use a cheaper alternative to formulate the shape of the skin.

Two properties of the human skin are of particular importance to achieve good visual quality of an animated character, which are volume preservation of the tissue under the skin; and the incompressibility of the skin itself. In the following we will develop equations that are able to capture the essence of these properties.

Volume Preservation. In the cross section of a wrinkle, the profile is similar to the curve given in Figure 6. At its rest pose the initial values of both parameters, $\mathrm{h}$ and $\mathrm{a}$, are given by the user. Assuming the skin was flat before a wrinkle was placed, the presence of the wrinkle ditches the skin, but the flesh is bulged at the sides.

We propose a piecewise polynomial function, called the shape function, to represent the two sections illustrated by the profile curve in Figure 6 . The two sections are $[0,2 a]$ and $[2 a, A]$. Both connect smoothly at $x=2 a$ when the shape function peaks. From Figure 6 , the constraints for the first section are that the curve passes three points $(0,-h),(a, 0)$ and $(2 a, h / 8)$, and satisfies $f^{\prime}(2 a)=0$. Thus the shape function for the first section is given by:

$$
f(x)=\frac{5 h}{32 a^{3}} x^{3}-\frac{29 h}{32 a^{2}} x^{2}+\frac{7 h}{4 a} x-h x \in[0,2 a]
$$

For the second section $[2 a, A]$, the shape function is developed from the cubic Hermite interpolation method, where unknown A is to be determined:

$$
f(x)=\frac{h}{4(A-2 a)^{3}} x^{3}-\frac{3 h(A+2 a)}{8(A-2 a)^{3}} x^{2}+\frac{3 h A a}{2(A-2 a)^{3}} x-\frac{-h A^{3}+6 h A^{2} a}{8(A-2 a)^{3}} x \in[0,2 a]
$$

In order to preserve the volume during deformation, the following condition is to be met:

$$
\int_{0}^{A} f(x) d x=0
$$

This gives:

$$
\int_{0}^{A} f(x) d x=\int_{0}^{2 a} f(x) d x+\int_{2 a}^{A} f(x) d x=-\frac{7 h a}{24}+\frac{h(A-2 a)}{16}=\frac{h(-20 a+3 A)}{48}=0
$$

Solving this integral equation, we obtain:

$$
A=\frac{20}{3} a
$$

Substituting Eq. (5) back into Eq. (2), the function of the second section arrives. In fact, parameter $A$ can also be controlled by the user if this is the user chooses to do. In that case, the volume may vary to suit the intended design of the skin shape. 


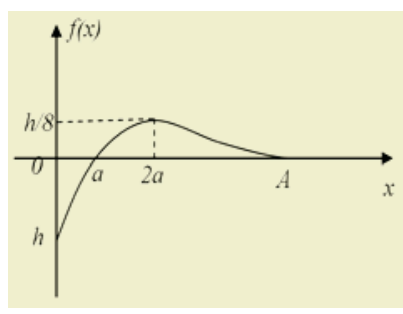

Fig. 6. Wrinkle shape function

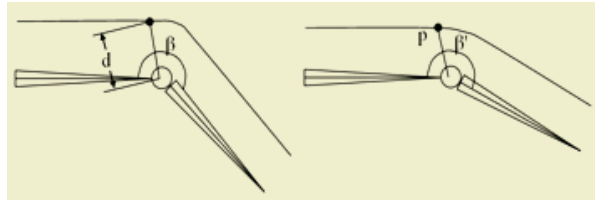

Fig. 7. Skeleton driven-animation

Incompressibility of Skin. Incompressibility is a different property to that of volume preservation. This property suggests that the length of the skin remains constant during animation. Compared with the former, this property is of more significance in visual realism. Linking with the shape function, this condition can be expressed by the following integral equation:

$$
G(h, a)=\int_{0}^{A} \sqrt{1+\left[f^{\prime}(x)\right]^{2}} d x=C
$$

where $C$ is a constant, which is calculated at the rest pose of the animated model. Since this equation involves two parameters $a$ and $h$, once the length $C$ is known, both parameters become mutually dependent of each other. Animating the model deforms the wrinkle profile, this further leads to a new value for parameter $a$ (how to compute the value of $a$ is explained at the next subsection). Thus Eq. (6) reduces to $G(h)=C$. This equation is not usually solvable analytically, but can be solved reasonably straightforwardly using a numerical method, such as the Newton method. This produces a new depth parameter $h$ for the new pose. As this is a one-dimensional problem, the resolution is fast. Real-time performance is achieved for all our experiments.

\subsection{Wrinkle Animation}

Skeleton-driven animation is the most popular technique for character animation, which we use here as the driving action for wrinkle animation. That said, when the skeleton moves, the character model, such as a hand model, together with the wrinkles on the skin surface is animated. What we are trying to work out is how such skeleton-driven animation is related to the shape change of the skin, and in turn to the change of the wrinkling parameters, $a$ and $h$, in our case.

Most hand joints have one dominating rotational degree of freedom (DOF), such as the fingers and palm. In fact, even for joints with a complex combination of angular movements, at an instant, it is reasonable to regard the rotational motion as being of one DOF, perhaps in an arbitrary orientation. This observation is useful in simplifying the animation process.

Let us look at the wrinkles around a finger joint, where the joint angle acts as the driving parameter. If it bends, the back of the finger stretches and the 
inside surface shrinks, which makes the wrinkle around the joint more or less prominent. Figure 7 shows the skeleton and skin surface around a joint. $P$ is a point on the wrinkle line, $d$ is the distance from the point to the joint centre, $\beta$ is the joint angle at the rest pose. When the joint rotates to angle $\beta^{\prime}$, the length of the skin surface is shortened and the shrinkage can be computed by:

$$
S=d * \frac{\beta^{\prime}}{\beta}
$$

Here $S$ indicates the amount of shortening of the skin relative to its original length. $S=1$ means it is the rest pose, $S>1$ suggests the skin expands, and $S<1$ signals the skin shrinks. Thus a corresponding new value of $a$ is given by:

$$
a=S * a_{0}
$$

where $a_{0}$ is the parameter at its rest pose.

With a we can compute a new value of $h$ using Eq. (6), if accuracy is important. Otherwise we can simply approximate the shape function curve between $[0, a]$ by a straight line whose length is kept constant. This results in a simpler formula for the depth $h$ :

$$
h=\left\{\begin{array}{cl}
\sqrt{h_{0}^{2}+\left(1-s^{2}\right) a_{0}^{2}} & s<\sqrt{1+\frac{h_{0}^{2}}{a_{0}^{2}}} \\
0 & s \geq \sqrt{1+\frac{h_{0}^{2}}{a_{0}^{2}}}
\end{array}\right.
$$

where $h_{0}$ denotes the parameter at the rest pose. This is reasonable, as when the skin exceeds the length of the skin when being stretched, the depth of the wrinkle will become zero. When the skin shrinks, the depth will increase reaching the maximum depth of $\sqrt{h_{0}^{2}+a_{0}^{2}}$.

Figure $8(\mathrm{a})$ shows the animation of a finger and the wrinkles on the back surface. Figure 8 (b) demonstrates the wrinkles generated on the palm and at the joints of the fingers.

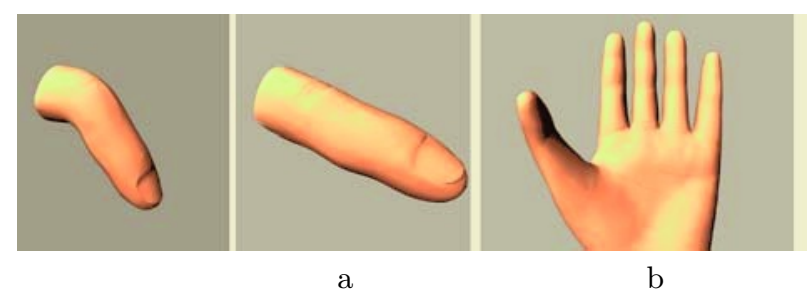

Fig. 8. Application of wrinkles: (a) wrinkled finger model (b)Wrinkles on a hand model

\section{Conclusion}

Wrinkles are important visual features on the skin of an animated human and creature character. In this paper, we have presented a new method for the gener- 
ation of hand wrinkles quickly and realistically. This method works in the following steps. Wrinkle lines are given at the first step, which are to catch the intent of the animator. Such wrinkle lines can be either manually drawn or scanned in. In the second step, a deformation region is defined for a group of closely laid wrinkle lines. Local meshes within the deformation regions are produced and merged with the mesh of the model. Compared with other existing techniques, our method is cheaper and needs a smaller number of vertices in the deformation region. A wrinkle shape function is derived in the third step, where both volume preservation and skin incompressibility are taken into account. Due to the exploitation of these two properties, our method is able to produce visually realistic results without using full scale physics. In the final step, the wrinkles are animated together with the hand model, where key parameters of the wrinkle shape function are determined at each pose.

Although our method uses the hand model explicitly, it is applicable also to other parts of the body where the skin is deformed by underlying skeleton joints.

\section{Acknowledgements}

This research is funded by the AHRB grant B/RG/AN5263/APN12727. The authors would also like to gratefully acknowledge Martin Held for providing us the code to generate Voronoi diagrams. We are grateful for the donation of the Maya licenses from Alias.

\section{References}

1. M.L. Viaud, H.Y.: Facial Animation with Wrinkles. Proc. Eurographics workshop on Animation and Simulation. (1992).

2. Bando, Y.T.U., Japan; Kuratate, T.; Nishita, T.: A simple method for modeling wrinkles on human skin. Proceedings 10th Pacific Conference on Computer Graphics and Applications. (2002)

3. Larboulette, C. and M.-P. Cani: Real-Time Dynamic Wrinkles, Computer Graphics International. (2004) IEEE Computer Society Press.

4. Wu, Y., N.M. Thalmann, and D. Thalmann: A plastic-visco-elastic model for wrinkles in facial animation and skin aging, Proceeding of the second Pacific conference on Fundamentals of computer graphics. (1994) 201-213.

5. Yin Wu, P.K., Laurent Moccozet, Nadia Magnenat-Thalmann, Simulating wrinkles and skin aging. The Visual Computer, 15(4)(1999) 183 - 198.

6. Boissieux, L.K.; Thalmann, N.M.; Kalra, P.: Simulation of skin aging and wrinkles with cosmetics insight. in Computer Animation and Simulation, Eurographics, (2000).

7. Held, M., VRONI: An engineering approach to the reliable and efficient computation of Voronoi diagrams of points and line segments. Computational Geometry, 18(2)(2001) 95-123. 